\title{
Dialisi a casa? Sì, grazie
}

\section{Gianna d'Adamo}

Nefrologa, Roma

\begin{abstract}
Dialysis at home? Yes thanks
Home dialysis is reported to provide similar or better survival than in center hemodialysis but few comparisons of outcomes with home hemodialysis (HHD) versus peritoneal dialysis (PD) exist. New insights about major clinical outcomes on HHD and PD were reported and compared in three large studies based on ANZDATA and USRDS data and published in 2015. HHD was associated with lower mortality and hospitalization rates. The outcomes of PD were better in new dialysis patients but modality failure was high.

Today more than ever, people with chronic kidney disease must be promptly and accurately informed about the chance to do home dialysis and to choose between HHD and PD.
\end{abstract}

Keywords: Home dialysis, Home hemodialysis, Outcomes, Peritoneal dialysis

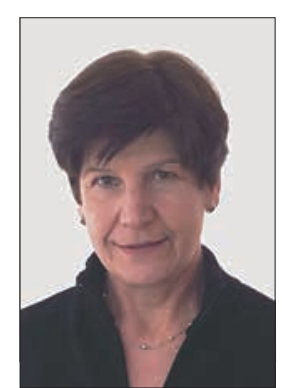

L'utilizzo e i preconcetti nei confronti della dialisi peritoneale (PD) sono invariati in Italia da anni. Invece, l'interesse nei confronti dell'emodialisi domiciliare (HHD) è tornato a crescere, favorito dalla disponibilità di un'apparecchiatura che semplifica l'esecuzione della terapia a domicilio. All'HHD si attribuiscono gli stessi vantaggi della PD rispetto all'emodialisi in centro (CHD): maggiore autonomia e migliore qualità di vita per il paziente e minor costo del trattamento (1).

Quest'anno sono stati pubblicati tre importanti lavori che confrontano con metodologia rigorosa i risultati clinici ottenuti con le due tecniche di dialisi domiciliare in casistiche molto numerose.

Lo studio retrospettivo di Suri et al. è condotto su dati del registro statunitense USRDS e riguarda una coorte costituita soprattutto da pazienti prevalenti con inizio del trattamento domiciliare tra il 2004 e il 2009. Riporta un tasso di ospedalizzazione significativamente più basso in HHD quotidiana che in PD (0.93 vs 1.35/anno paziente) e una minore durata dei ricoveri (5.2 vs $9.2 \mathrm{gg} / \mathrm{anno}$ paziente) (2). L'ulteriore confronto di pazienti in HHD quotidiana e pazienti trattati con CHD non

Accepted: October 13, 2015

Published online: November 19, 2015

Indirizzo per la corrispondenza:

Dr.ssa Gianna d'Adamo

Nefrologa, Roma

giannadadamo@gmail.com mostra una differenza significativa dei tassi di ricovero (2).

Nello studio di Nadeau-Fredette et al. sui pazienti incidenti (in dialisi domiciliare a 90 giorni dall'inizio della terapia sostitutiva) del registro ANZDATA di Australia e Nuova Zelanda tra il 2000 e il 2012, I'HHD si associa a una maggiore sopravvivenza dei pazienti (98\%, 95\% e $85 \%$ a 1, 2 e a 5 anni) rispetto alla PD (89\%, $76 \%$ e $44 \%$ a 1,2 e a 5 anni) (3). Sui dati dello stesso registro era stato rilevato in precedenza che i trattamenti a domicilio si associavano a una sopravvivenza migliore rispetto a quella riscontrata nei trattamenti effettuati in Centro dialisi: in PD il vantaggio era modesto e limitato ai primi 3 anni di trattamento, mentre, in HHD, era superiore e protratto nel tempo (4).

Nello studio di Weinhandl et al. su dati dell'USRDS, in una coorte costituita soprattutto da pazienti prevalenti con inizio del trattamento domiciliare tra il 2007 e il 2010, il rischio di morte e il rischio di ospedalizzazione sono inferiori in HHD quotidiana che in PD, rispettivamente del $20 \%$ e dell' $8 \%$. I rischi delle due tecniche sono, invece, simili nei sottogruppi di pazienti che hanno cominciato la dialisi a domicilio entro 6 mesi dall'inizio del trattamento sostitutivo (5).

In tutti e tre questi studi $(2,3,5)$, il fallimento della tecnica con passaggio all'HD in centro dialisi è significativamente minore in HHD, indipendentemente dalla durata della terapia sostitutiva.

I dati mostrano, quindi, che l'HHD offre la prospettiva di poter dializzare più a lungo a casa propria e confermano specificità della PD note ormai da molti anni: 1) i risultati migliori si ottengono all'inizio della terapia dialitica; 2) il dropout della tecnica è molto alto.

Questi risultati appartengono a realtà molto diverse da quella italiana sia come geografia che come sistemi sanitari. Inoltre, mancano alcune informazioni che vorremmo co- 
noscere, come, per esempio, i motivi della scelta del tipo di trattamento, la diuresi residua, lo schema dialitico e così via. Tuttavia, viste la numerosità delle casistiche e l'accuratezza delle analisi statistiche, l'interesse di questi studi è molto grande ed è fin troppo scontato l'auspicio di avere presto dati che confrontino i risultati della PD e della "rinata" HHD anche nella nostra realtà.

Nell'attesa, non possono, comunque, sussistere dubbi sul dovere di informare le persone con malattia renale cronica della possibilità di eseguire la dialisi a domicilio e di poter scegliere tra HHD e PD in relazione alla propria situazione clinica e sociale.

Per l'HHD è sempre richiesta la presenza di un caregiver opportunamente formato e il periodo di training è più lungo. La PD si esegue da soli e può essere un eccellente "ponte" per il trapianto che, il più delle volte, non può essere realizzato all'inizio della terapia sostitutiva.

La tempestività e la completezza dell'informazione su tutte le terapie sostitutive sono la premessa indispensabile perché ciascuno possa accedere alla soluzione terapeutica che è migliore per lui e perché le organizzazioni possano utilizzare al meglio le risorse disponibili.

\section{Disclosures}

Financial support: No financial support was received for this submission. Conflict of interest: The author has no conflict of interest.

\section{Bibliografia}

1. Abdel-Kader K, Unruh ML. Benefits of short daily home hemodialysis in the FREEDOM Study: is it about person, place, time, or treatment? Kidney Int 2012;82:511-3.

2. Suri RS, Li L, Nesrallah GE. The risk of hospitalization and modality failure with home dialysis. Kidney Int 2015;88:360-8.

3. Nadeau-Fredette AC, Hawley CM, Pascoe EM, et al. An Incident Cohort Study Comparing Survival on Home Hemodialysis and Peritoneal Dialysis (Australia and New Zealand Dialysis and Transplantation Registry). Clin J Am Soc Nephrol 2015;10: 1397-407.

4. Marshall MR, Walker RC, Polkinghorne KR, Lynn KL. Survival on home dialysis in New Zealand. PLoS ONE 2014;9:e96847.

5. WeinhandlED, Gilbertson DT, Collins AJ. Mortality, Hospitalization, and Technique Failure in Daily Home Hemodialysis and Matched Peritoneal Dialysis Patients: A Matched Cohort Study. Am J Kidney Dis 2015; Aug 26. pii: S0272-6386(15)01018-5. doi: 10.1053/j.ajkd.2015.07.014. [Epub ahead of print]. 